\title{
Comportamiento de los sedimentos fluviales en Honduras. Rendimiento y pérdida de suelo Zona Central y Oriental
}

\author{
Roberto Fredy Ávalos Lingan*
}

\section{RESUMEN}

El Ministerio de Recursos Naturales y Ambiente hasta 1984 manejaba una fuerte campaña de medición de sedimento en suspensión en sus principales estaciones hidrométricas. Por otra parte la Empresa Nacional de Energía Eléctrica (ENEE) manejaba también hasta los años 90 una fuerte medición de sedimento en las cuencas de su interés. La ENEE en los años 2000 a retomado dichas mediciones. Toda la información recolectada, a la fecha, presenta mucha discontinuidad en el tiempo y en frecuencia de medición. La información rescatable ha sido pocas veces investigada; al grado que los estudios sedimentológicos de los proyectos hidroeléctricos se basan en criterio de rendimiento y pérdida de suelo, según la experiencia de los consultores.

De lo mencionado se creyó necesario investigar, con la información disponible, los rendimientos y pérdida de suelo para la zona central y oriental del país. Esto permitirá disponer de alguna base científica para evaluar los valores utilizados por los consultores.

La hipótesis que el rango de rendimiento se encuentra entre 500 y 1000 (Ton/año/km²) es aceptada como resultado de la presente investigación.

Palabras Clave: Sedimento, Sedimento en Suspensión, Sedimento de fondo, Muestreo Integral, Caudal Líquido, Caudal Sólido, Relación, Rendimiento y Pérdida de Suelo.

\section{ABSTRACT}

Up to 1984 the Ministry of Natural and Environmental Resources carried and intensive measurement campaing of suspending sludge in its main hydrometric stations. On the other hand up to the 90's the Electrical National Company (ENEE)

\footnotetext{
*Roberto Fredy Ávalos Lingan

Facultad de Ingeniería, Universidad Nacional Autónoma de Honduras
} 
also carried an intensive sludge measurement in the water basins of interest. By the year 2000 the (ENEE) has collected such measurements. All the information gathered up to now presents discontinuity in time and in measurement frequency. Recoverable information has been investigated a few times to the point sedimentological studies of the hydroelectrical projects are based on the criterion of the usefulness and loss of the ground according to the consultant's experience.

As mentioned before it was necessary to investigate with the available information the usefulness and loss of the ground for the central and western zones of the country. This will provide to access some scientific evidence to evaluate the figures used by the consultants.

The hypothesis that the usefulness ranges between 500 and 1000 (ton/year $/ \mathrm{km}^{2}$ is accepted as results in the present investigation.

Keywords: Sludge, suspending sludge, bottom sludge, integral sampling, liquid volume, solid volume, relation, ground usefulness and loss. 


\section{INTRODUCCIÓN}

Cada inicio del período lluvioso se observa en las diferentes cuencas hidrográficas del país que las aguas de los ríos y quebradas transportan agua con una fuerte turbidez. Dependiendo esto, principalmente, de la lluvia (cantidad, intensidad y duración), del tipo y estado del suelo, de la cobertura vegetal y de la morfología de la cuenca. De acuerdo a la publicación realizada por La Secretaría de Recursos Naturales y Ambiente (Balance Hídrico de Honduras, 2004), en esta se menciona que la lluvia promedio en Honduras es alta comparado con la evapotranspiración

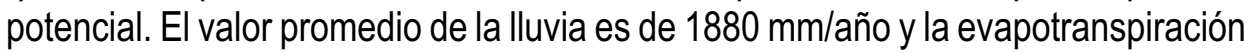
potencial de $1315 \mathrm{~mm} / a n ̃ o$. Calificándole como un país de clima húmedo y subhúmedo con una fuerte riqueza hídrica.

La lluvia es el principal agente en la producción de sedimento en la cuenca y los caudales en los cursos de agua en la erosión del lecho. Tomando en cuenta la situación actual de las cuencas del punto de vista de cobertura vegetal y constitución y estado del suelo, y lo mencionado en el párrafo anterior, el territorio hondureño se encuentra sometido a un fuerte potencial de erosión.

El objetivo de la presente investigación es determinar, a partir de la información disponible, los rendimientos de sedimento en ton/año $/ \mathrm{km}^{2}$ y la pérdida de suelo en $\mathrm{mm} / \mathrm{año}$; para la zona central y oriental del país.

Como resultado podemos mencionar que la parte oriental de país tiene un rendimiento de 685.586 ton/año $/ \mathrm{km}^{2}$ y una pérdida de suelo de $0.857 \mathrm{~mm} / \mathrm{año} \mathrm{y} \mathrm{la}$ parte central de 843.344 ton/año $/ \mathrm{km}^{2}$ y una pérdida de suelo de $1.054 \mathrm{~mm} / \mathrm{año}$.

\section{ANTECEDENTE}

De acuerdo a la información obtenida se sabe que desde lo años 60 el Departamento de Hidrología y Climatología de la Dirección de Recursos Hídricos (DGRH) del Ministerio de Recursos Naturales y Ambiente realizaba muestreos de sedimento en los principales ríos del país y que mediante su laboratorio de sedimento, de ese tiempo, obtenían las concentraciones y la producción de sedimento. Aparentemente esto duró hasta mediados de los años 84; información de sedimento antes de esos años no fue posible obtener.

Por otra parte, la Empresa Nacional de Energía Eléctrica (ENEE) también desde los años 60 inició muestreos de sedimentos integral y puntual en sus estaciones donde 
se proyectaba una central hidroeléctrica. Disponía de un laboratorio de sedimento para la medición de concentraciones. A la fecha actual el laboratorio se ha modernizado; no solamente para concentraciones de sedimento, sino también de calidad de agua y otras variables más.

A través del tiempo los muestreos de sedimento en las estaciones hidrométrica de la ENEE disminuyeron; pero actualmente se ha retomado el plan de operaciones de muestreo. Cabe mencionar que ambas instituciones gubernamentales solamente han medido o miden sedimento en suspensión.

En el Estudio de Factibilidad del Proyecto El Cajón (1973), Volumen 2 Hidrología, la Empresa Motors-Columbus de Suiza tomaron los datos de sedimento disponibles a esa fecha del río Humuya y a su vez ellos también realizaron algunos muestreos. Llegando finalmente a determinar un rendimiento de 733.25 ton $/ a n ̃ o / \mathrm{km}^{2}$.

La ENEE en 1987 realizó un análisis de sedimento de sus estaciones hidrométricas que disponían mayor información. Para lo cual aplicó el método del Bureau of Reclamation, considerando las curvas de sedimento ajustadas a las líneas de concentración establecidas por la ENEE y las curvas de duración de los caudales diarios. Lamentablemente no se pudo disponer de los datos ni del informe; pero si de algunas curvas de sedimentos.

En mayo de 1991 la Consultora alemana LAHMEYER dentro de los estudios de los proyectos hidroeléctricos Remolino y Sico II, determinó para el río Patuca en la estación hidrométrica Cayetano el rendimiento de 133.0 ton $/ a n ̃ o / \mathrm{km}^{2}$. Calificando este resultado como muy bajo debido básicamente a la calidad de la información existente y a su discontinuidad. Finalmente esta Empresa trabajó con rangos de rendimiento de 500 a 900 ton $/ a n ̃ o / \mathrm{km}^{2}$.

\section{MARCO CONCEPTUAL}

A pesar que hay algo de información de sedimento en suspensión muestreados por las instituciones anteriormente mencionadas; cuyos planes de muestreos quizás no fueron realizados bajo ningún criterio científico; pero es la información que actualmente se dispone. Ya que a la fecha no es posible determinar en forma exacta los rendimientos y pérdida de suelo para las diferentes cuencas del país; queda la pregunta en qué rango se puede encontrar los rendimiento y pérdida de suelo en Honduras?.

Son los proyectos hidroeléctricos que han hecho realidad las mediciones de 
sedimento que actualmente existe en el país. Básicamente esto es utilizado para determinar el volumen muerto de los embalses. El especialista y el diseñador se ponen de acuerdo para discutir los resultados y proponer un valor razonable. Conocer las pérdidas de suelo de las cuencas y de los cursos de agua es importante para el desarrollo sostenible y sustentable del país; recordemos que son en las primeras capas del suelo donde se encuentra la riqueza agrícola y no mencionar sus capacidades físicas que se ponen en riesgo. Esto permitirá establecer científicamente planes operativos urgentes para la conservación de los suelos.

\section{HIPÓTESIS}

La hipótesis a probar es: Para la zona Central y Oriental los rendimientos se encuentran entre 500 y 1000 ton $/ a n ̃ o / \mathrm{km}^{2}$.

\section{METODOLOGÍA}

En base a la información disponible las muestras para la zona central es representada, en esta investigación, por la información disponible de los ríos Malapa, Guacamaya, Maragua, de sus estaciones hidrométricas correspondientes; tomando consideración únicamente las muestras obtenidas por muestreo integral. Para el caso de la zona oriental se pudo contar con los datos de sedimento de muestreo integral del río Patuca justo después de la unión de los ríos Guayape y Guayambre en la estación hidrométrica Cayetano. Cabe mencionar que las estaciones hidrométricas utilizadas pertenecen y son manejadas por la ENEE. El Plano 1 muestra la ubicación de los sitios de muestreo.

La calidad de la realización de los muestreos y los análisis de laboratorio está regida por normas internacionales, en especial, por la Organización Mundial Meteorológica y por parte de los fabricantes de equipo. En cuanto a la frecuencia de muestreo las normas dicen que hay que realizarlos durante todo el año; pero con una frecuencia mayor en los meses lluviosos; debidos a que son los tiempos de mayor sedimento. La cantidad fijada de cuantos muestreos hay que realizar en cada mes/meses seco o lluvioso no está bien establecida. La ENEE trata de cumplir con estas normas, cuyo cumplimiento en cuanto a la frecuencia están sujetas a circunstancias y planes propios de esa institución; como por ejemplo prioridades.

Cabe señalar que no se dispone de una serie con un número de datos uniformes de sedimento en suspensión en cada estación hidrométrica mencionada. Los análisis a aplicar serán realizados para el periodo disponible de cada una de ellas. Para 
determinar los rendimientos y pérdida de suelo de las cuencas con información disponible, la metodología consistirá en encontrar alguna relación entre los caudales líquidos QL ( $\left.\mathrm{m}^{3} / \mathrm{s}\right)$ y sólidos $Q S$ (ton/día) justo en el periodo en que se realizaron los muestreos. Esto permitirá encontrar una relación matemática que las relacione y después de esto, extrapolarla o interpolarla de acuerdo a la información de caudales líquidos diarios disponibles. El peso específico del sedimento en suspensión es considerado en esta investigación con un valor de 1.25 ton $/ \mathrm{m}^{3}$.

Plano 1

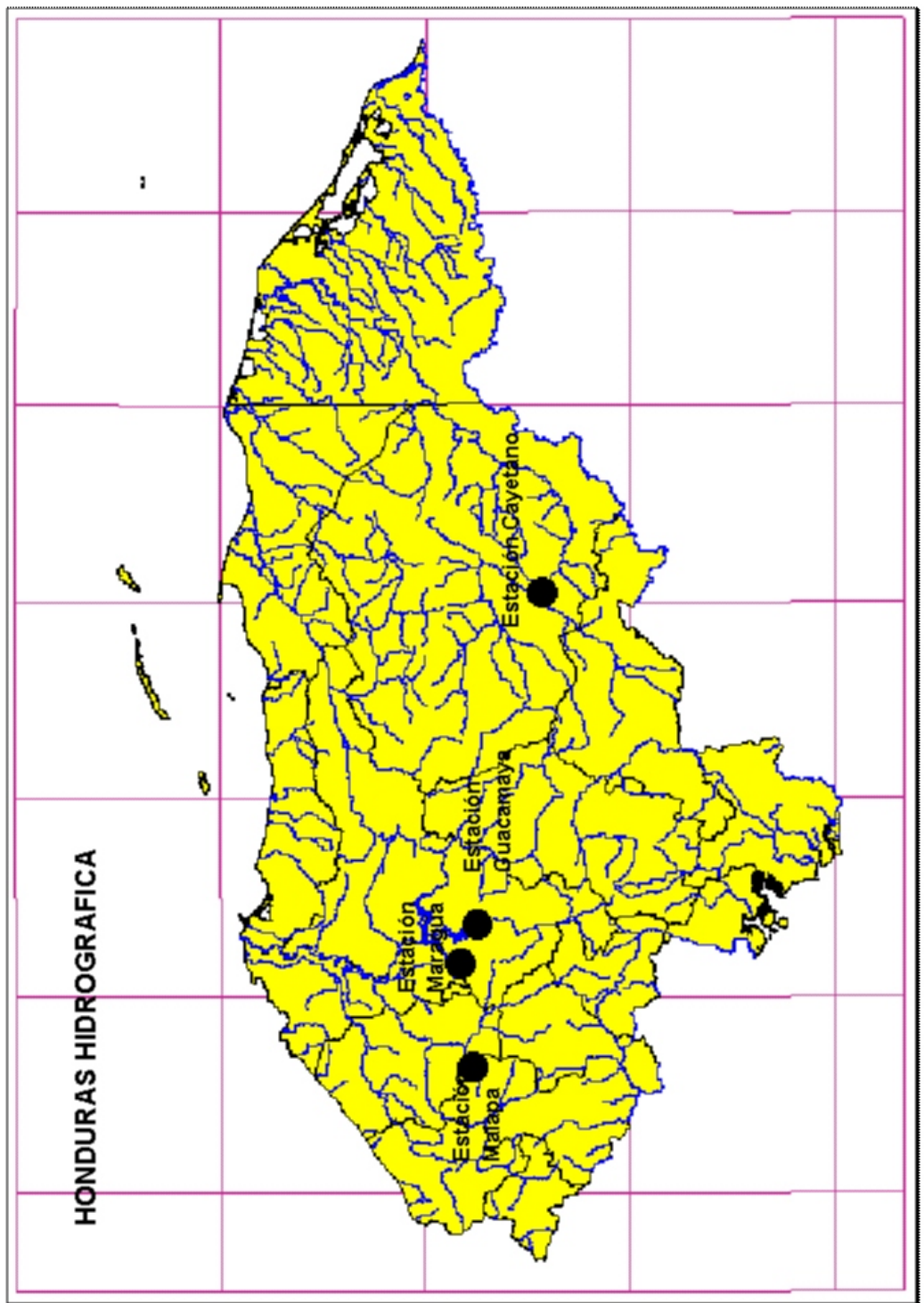




\section{APLICACIÓNYRESULTADOS}

\section{Generalidades}

La metodología fue aplicada a las estaciones hidrométricas de Cayetano Guacamaya, Malapa y Maragua. Se probó con cada una de ellas varios tipos de relación entre QL vs QS, a mencionar relación lineal, relación polinomial de diferente orden. Logarítmica potencial y exponencial. Los mejores ajustes se obtuvieron utilizando un ajuste potencial, es decir $Q S=c^{*} Q L^{\wedge} n$. Por consiguiente esta misma metodología fue aplicada a la información disponible de cada estación hidrométrica; permitiendo una uniformidad del tratamiento de la información y por supuesto una mejor interpretación de los resultados.

\section{Río Patuca}

Al sitio de la estación hidrométrica Patuca en Cayetano la cuenca correspondiente cubre un área hidrográfica de $10566.9 \mathrm{~km}^{2}$. La información recolectada de sedimento para esta investigación abarca el periodo de Marzo 2004 aAgosto 2007.

La relación encontrada entre $\mathrm{QL}\left(\mathrm{m}^{3} / \mathrm{s}\right)$ y QS (ton/día) es la siguiente:

$$
Q S=0.0199 * Q^{2.3902}
$$

El coeficiente de correlación determinado es de 0.94 .

La Figura 1 muestra el ploteo de la información en Log-Log y el trazado de la ecuación que relaciona ambas variables. Utilizando los caudales líquidos diarios para el periodo 2004 y 2006 y la ecuación encontrada, el promedio obtenido de los rendimientos es de 133.445 (ton $/ a n ̃ o / \mathrm{km}^{2}$ ) y lo concerniente a las pérdidas de suelo fue de $0.167 \mathrm{~mm} /$ año.

FIGURA 1: Estación Cayetano. Ploteo de QS vs QL

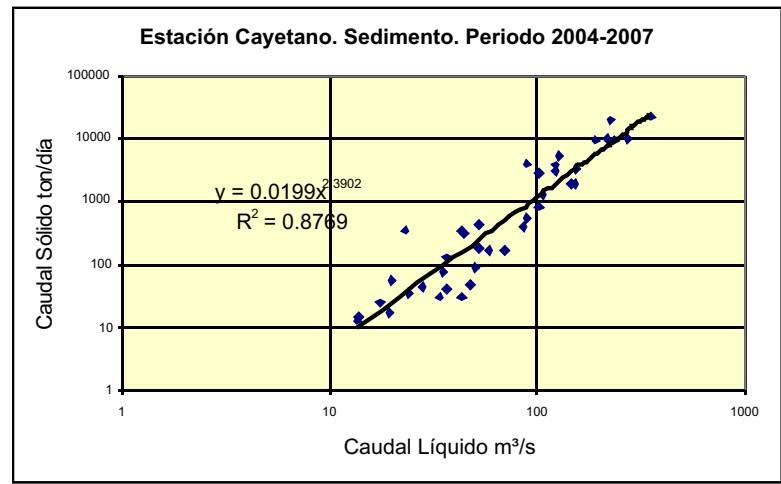


Como la estación hidrométrica de Cayetano tiene información de caudales líquidos desde 1973; la ecuación QS = f(QL) fue extrapolada de 1973 al 2006. Haciendo un total de datos de 33 años. El Cuadro 1 muestra los resultados obtenidos.

En el Cuadro 1 se observa los rendimientos y pérdida de suelo para cada año y también al final del cuadro un promedio y un total. El promedio corresponde a los sedimentos en suspensión y el total al sedimento total; es decir los sedimentos en suspensión más el de fondo.

De acuerdo al texto Diseño de Pequeñas Presas, publicado por el Bureau of Reclamation, se menciona que cuando no se dispone de información el sedimento de fondo, puede ser representado por el 10 al $15 \%$ del sedimento en suspensión, en nuestro caso consideramos un $15 \%$.

\section{Río Humuya}

Al sitio de la estación hidrométrica Humuya en Guacamaya la cuenca correspondiente cubre una cuenca hidrográfica de 2570.0 $\mathrm{km}^{2}$. La información recolectada para esta investigación abarca el periodo de Agosto 1992 a Noviembre 2002.

La relación encontrada entre QL $\left(\mathrm{m}^{3} / \mathrm{s}\right)$ y QS (ton/día) es la siguiente:

$$
Q S=0.0704^{*} Q L^{2.5492}
$$

Con un coeficiente de correlación de 0.90 .

La Figura 2 muestra el ploteo de la información en Log-Log y el trazado de la ecuación que relaciona ambas variables.

Para el periodo 19922002 el rendimiento obtenido fue de 700.368 ton $/ a n ̃ o / \mathrm{km}^{2} \mathrm{y}$ la pérdida de suelo de $0.875 \mathrm{~mm} / \mathrm{año}$.

Como se pudo disponer de caudales diarios de la estación hidrométrica de Guacamaya desde 1987 al 2006 la ecuación fue también aplicada para dicho periodo. El Cuadro 2 presenta los resultados. 


\begin{tabular}{|c|c|c|}
\hline \multirow{2}{*}{ Año } & Rendimiento & Pérdida Suelo \\
\hline & (ton/año/km²) & (mm/año) \\
\hline 1973 & 111.263 & 0.139 \\
\hline 1974 & 147.750 & 0.185 \\
\hline 1975 & 859.247 & 1.074 \\
\hline 1976 & 137.662 & 0.172 \\
\hline 1977 & 215.518 & 0.269 \\
\hline 1978 & 326.393 & 0.408 \\
\hline 1979 & 2284.035 & 2.855 \\
\hline 1980 & 554.592 & 0.693 \\
\hline 1981 & 372.186 & 0.465 \\
\hline 1982 & 623.205 & 0.779 \\
\hline 1983 & 301.777 & 0.377 \\
\hline 1984 & 304.238 & 0.380 \\
\hline 1985 & 70.835 & 0.089 \\
\hline 1986 & 527.349 & 0.659 \\
\hline 1987 & 407.178 & 0.509 \\
\hline 1988 & 635.954 & 0.795 \\
\hline 1989 & 260.183 & 0.325 \\
\hline 1990 & 314.737 & 0.393 \\
\hline 1991 & 721.555 & 0.902 \\
\hline 1992 & 291.764 & 0.365 \\
\hline 1993 & 1154.212 & 1.443 \\
\hline 1994 & 128.703 & 0.161 \\
\hline 1995 & 1409.723 & 1.762 \\
\hline 1996 & 396.404 & 0.496 \\
\hline 1997 & 394.449 & 0.493 \\
\hline 1998 & 5098.543 & 6.373 \\
\hline 1999 & 1463.812 & 1.830 \\
\hline 2000 & 74.677 & 0.093 \\
\hline 2001 & 24.651 & 0.031 \\
\hline 2002 & 141.861 & 0.177 \\
\hline 2003 & 114.705 & 0.143 \\
\hline 2004 & 69.254 & 0.087 \\
\hline 2005 & 282.806 & 0.354 \\
\hline 2006 & 48.285 & 0.060 \\
\hline Promedio & 596.162 & 0.745 \\
\hline S. Fondo(15\%) & & \\
\hline Total & 685.586 & 0.857 \\
\hline
\end{tabular}


FIGURA 2: Estación Guacamaya. Ploteo QS VS QL

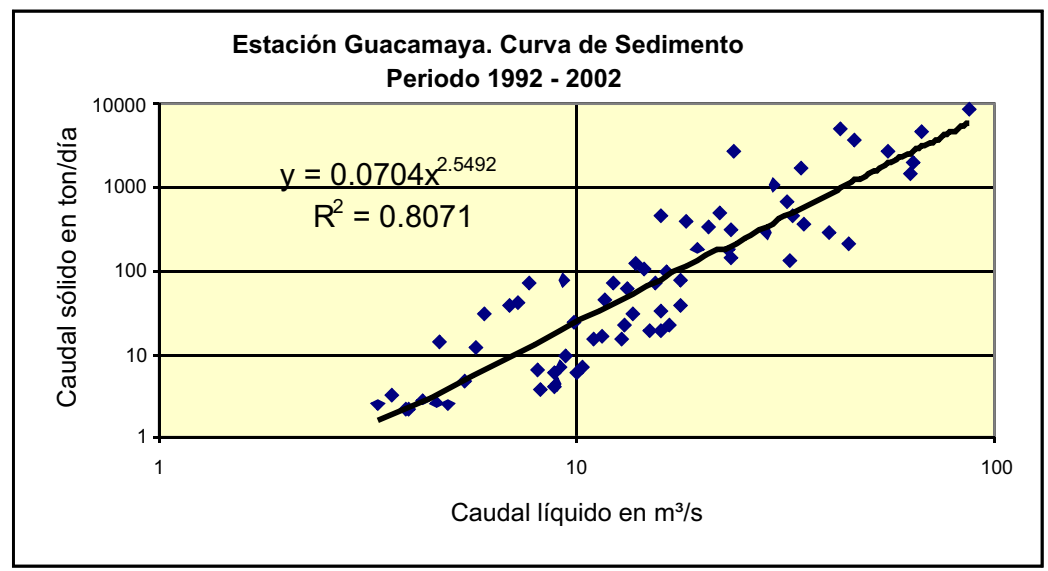

CUADRO 2: Río Humuya en la Estación Guacamaya. Rendimiento y Pérdida de Suelo

\begin{tabular}{|c|c|c|}
\hline \multirow{2}{*}{ Año } & Rendimiento & Pérdida Suelo \\
\hline & (ton/año/km²) & (mm/año) \\
\hline 1987 & 150.6035798 & 0.188254475 \\
\hline 1988 & 1554.783772 & 1.943479714 \\
\hline 1989 & 630.8049274 & 0.788506159 \\
\hline 1990 & 709.4182196 & 0.886772774 \\
\hline 1991 & 308.1359937 & 0.385169992 \\
\hline 1992 & 2038.584667 & 2.548230833 \\
\hline 1993 & 305.5494008 & 0.381936751 \\
\hline 1994 & 240.4778871 & 0.300597359 \\
\hline 1995 & 1791.775612 & 2.239719515 \\
\hline 1996 & 557.7196896 & 0.697149612 \\
\hline 1997 & 373.4576691 & 0.466822086 \\
\hline 1998 & 1800.001537 & 2.250001921 \\
\hline 2000 & 340.7901051 & 0.425987631 \\
\hline 2001 & 74.95226168 & 0.093690327 \\
\hline 2002 & 132.1481716 & 0.165185215 \\
\hline 2003 & 217.5991135 & 0.271998892 \\
\hline 2004 & 141.0399826 & 0.176299978 \\
\hline 2005 & 1608.406153 & 2.010507692 \\
\hline 2006 & 957.2654461 & 1.196581808 \\
\hline Promedio & 733.343 & 0.917 \\
\hline S. Fondo(15\%) & & \\
\hline Total & 843.344 & 1.054 \\
\hline
\end{tabular}




\section{Río Maragua}

Al sitio de la estación hidrométrica Maragua en Maragua la cuenca correspondiente cubre una cuenca hidrográfica de $252.3 \mathrm{~km}^{2}$. La información recolectada para esta investigación abarca el periodo de Agosto 1992 a Noviembre 2004.

La relación encontrada entre QL $\left(\mathrm{m}^{3} / \mathrm{s}\right)$ y QS (ton/día) es la siguiente:

$$
Q S=0.0888^{\star} Q L^{2.4966}
$$

Con un coeficiente de correlación de 0.933 .

La Figura 3 muestra el ploteo de la información en Log-Log y el trazado de la ecuación que relaciona ambas variables. Para el periodo 19922004 el rendimiento obtenido fue de 122.527 ton $/ a n ̃ o / \mathrm{km}^{2}$ y la pérdida de suelo de $0.153 \mathrm{~mm} / \mathrm{año}$.

Como se pudo disponer de caudales diarios de la estación hidrométrica de Maragua desde 1988 al 2006 la ecuación fue también aplicada para dicho periodo. El Cuadro 3 presentalos resultados.

FIGURA 3: Estación Maragua. Ploteo de QS vs QL

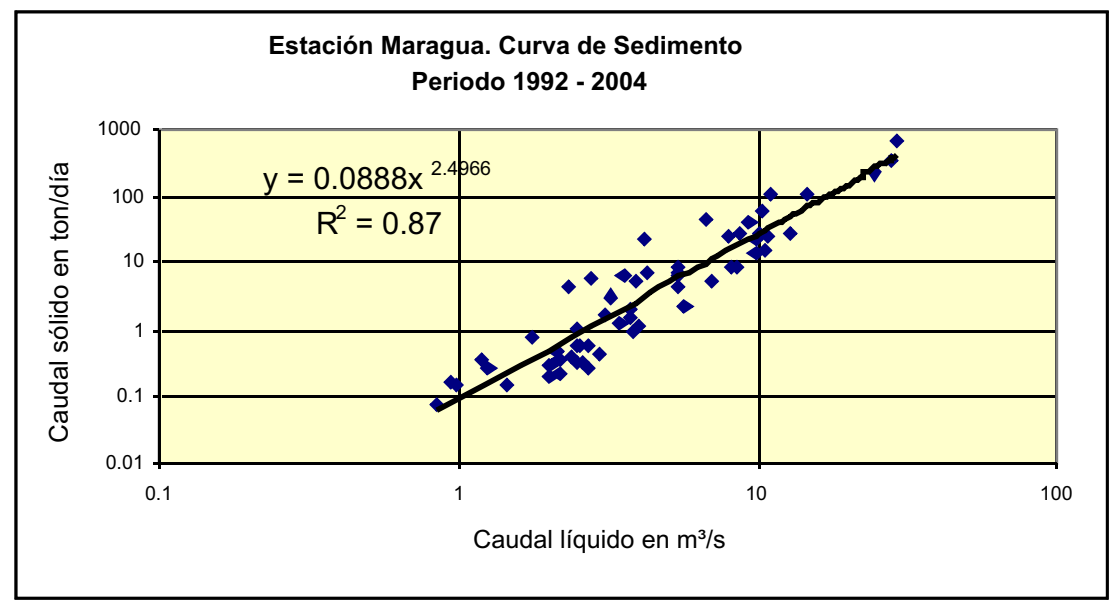




\section{CUADRO 3: Río Maragua en la estación Maragua. Rendimiento y Pérdida de Suelo}

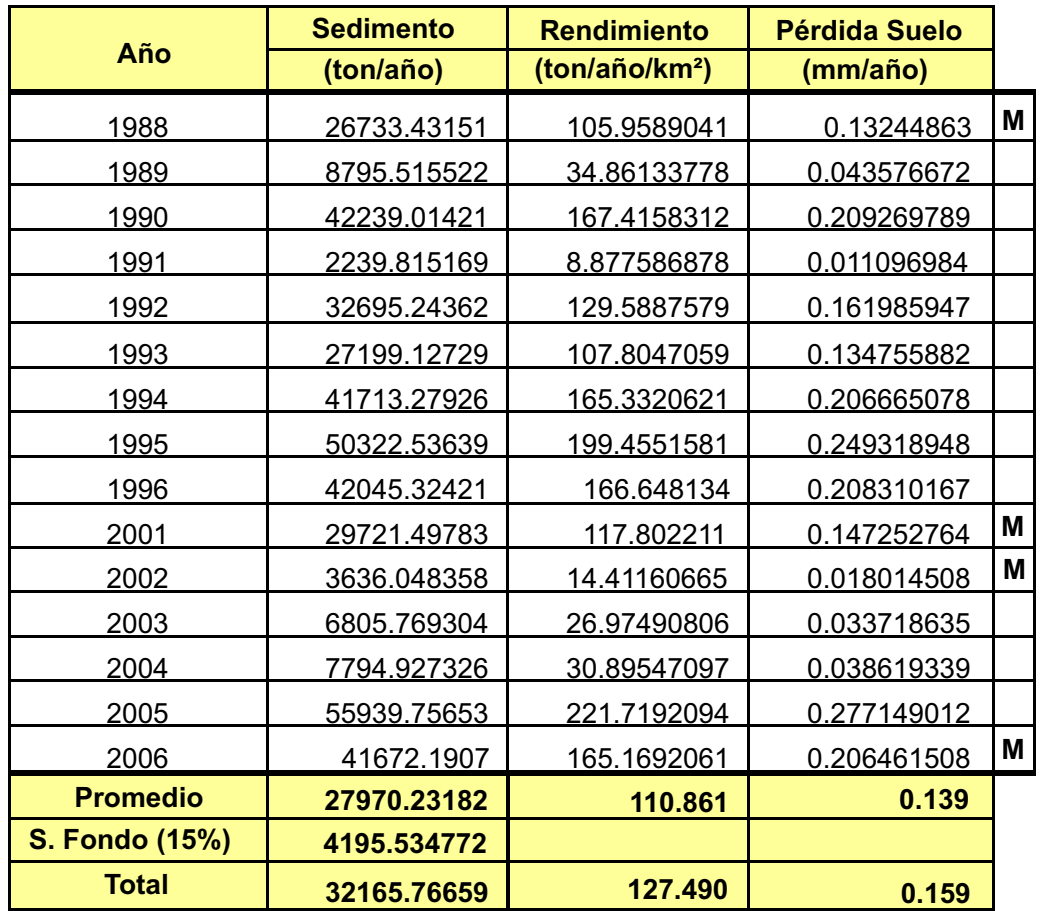

Nota

M Serie completada porque no tiene información en algunos meses del periodo seco.

\section{Río Malapa}

Al sitio de la estación hidrométrica Malapa en Malapa la cuenca correspondiente cubre una cuenca hidrográfica de $110.33 \mathrm{~km}^{2}$.

La información recolectada para esta investigación cubre el periodo de 1991 al 2006. La relación encontrada entre QL $\left(\mathrm{m}^{3} / \mathrm{s}\right)$ y QS (ton/día) es la siguiente:

$$
Q S=0.6833^{*} Q L^{1.7487}
$$

Con un coeficiente de correlación de 0.90 .

La Figura 4 muestra el ploteo de la información en Log-Log y el trazado de la ecuación que relaciona ambas variables. Para el periodo 1991- 2006 el 
rendimiento obtenido fue de 71.509 ton/año $/ \mathrm{km}^{2}$ y la pérdida de suelo de 0.089 $\mathrm{mm} / \mathrm{año}$.

Como se pudo disponer de caudales diarios de la estación hidrométrica de Maragua desde 1988 al 2006 la ecuación fue también aplicada para dicho periodo. El Cuadro 4 presenta los resultados.

FIGURA 4: Rio Malapa. Ploteo QS vs QL

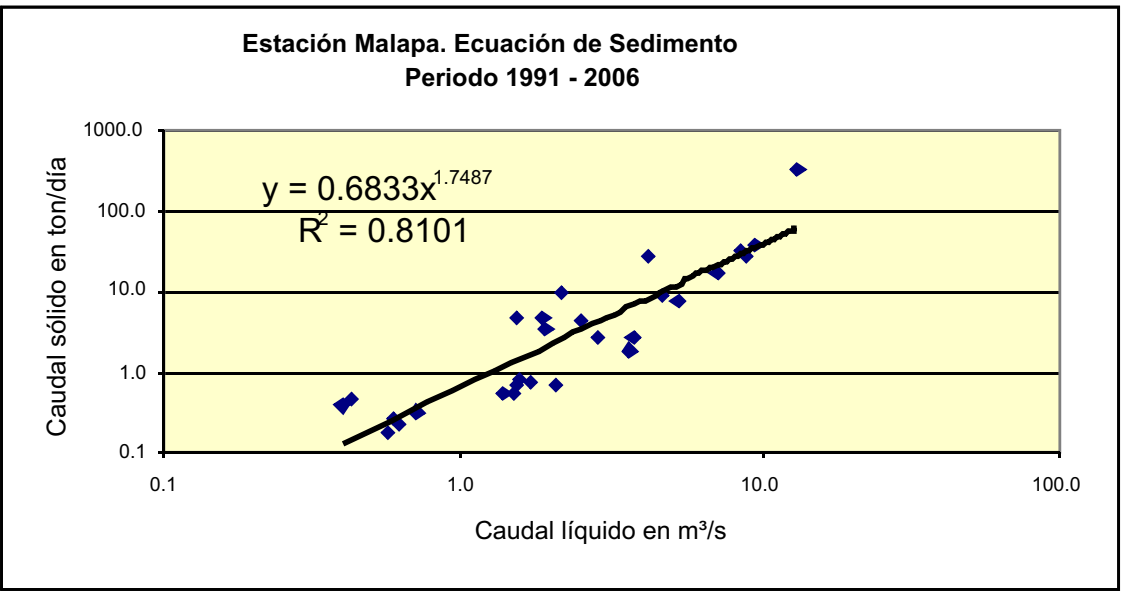

\section{ANÁLISIS E INTERPRETACIÓN}

Debido que no se tiene una serie larga de información de sedimento en suspensión se trató de utilizar toda la información que fue posible obtener; esto evitó seleccionar un período común de análisis para cada estación investigada.

A parte de esto se pudo observar que por lo general la mayor cantidad de información es obtenida en los meses secos. Provocando esto una subestimación de los rendimientos (ton/año/ $\mathrm{km}^{2}$ ) y pérdida de suelo (mm/año); por ejemplo los casos más notorios son los resultados obtenidos en los ríos de Malapa (afluente al embalse de la hidroeléctrica El Nispero) y Maragua (afluente al embalse El Cajón). De averiguaciones en la ENEE se dice que estos ríos tienen un mayor rendimiento y pérdida de suelo. 


\section{CUADRO 4: Río Malapa en la estación Malapa. Rendimiento y Pérdida de Suelo}

\begin{tabular}{|c|c|c|c|c|}
\hline \multirow{2}{*}{ Año } & Sedimento & Meses & Pérdida Suelo & Rendimiento \\
\hline & (ton/año) & Faltantes & (mm/año) & (ton/año/km²) \\
\hline 1988 & 10514.009 & & 0.119 & 95.296 \\
\hline 1989 & 11199.178 & & 0.127 & 101.506 \\
\hline 1990 & 10222.571 & & 0.116 & 92.654 \\
\hline 1991 & 5494.083 & & 0.062 & 49.797 \\
\hline 1992 & 5588.366 & & 0.063 & 50.651 \\
\hline 1993 & 4491.765 & & 0.051 & 40.712 \\
\hline 1994 & 2872.759 & & 0.033 & 26.038 \\
\hline 1995 & 24006.000 & & 0.272 & 217.584 \\
\hline 1996 & 10724.673 & & 0.122 & 97.205 \\
\hline 1997 & 4575.768 & 10,11 y 12 & & \\
\hline \multicolumn{5}{|l|}{1998} \\
\hline \multicolumn{5}{|l|}{1999} \\
\hline \multicolumn{5}{|l|}{2000} \\
\hline \multicolumn{5}{|l|}{2001} \\
\hline \multicolumn{5}{|l|}{2002} \\
\hline 2003 & 3666.668 & 1,2 y 3 & & \\
\hline 2004 & 485.531 & del 7 al 12 & & \\
\hline 2005 & 4621.396 & $1.2345 \times 8$ & & \\
\hline 2006 & 8938.989 & & 0.101 & 81.020 \\
\hline Suma & 94052.392 & & & \\
\hline Promedio & 9405.239 & & 0.107 & 85.246 \\
\hline S. Fondo(10\%) & 940.524 & & & \\
\hline Total & 10345.763 & & 0.117 & 93.771 \\
\hline
\end{tabular}

El extrapolar las relaciones hacia años anteriores no nos asegura que el comportamiento de los sedimentos sean iguales a los años recientes. Pero si sería interesante conocer la evolución de los rendimientos a través de periodos anteriores.

Si comparamos las relaciones obtenidas $Q S=f(Q L)$ (ver Figura 5) se observa que las tendencias entre Guacamaya y Maragua son muy parecidas y de igual forma la de Cayetano se asemeja a Malapa. En el caso de Maragua y Guacamaya ambos son afluentes al embalse El Cajón. La coincidencia entre Cayetano y Malapa aparentemente es estadístico. 
De acuerdo a la información disponible las estaciones de Guacamaya y Cayetano son las que presentaron mayor número de información y además, cubren cuencas de mayor amplitud que las otras dos. Al estado actual de la información y para esta investigación los resultados obtenidos en Cayetano y Guacamaya pueden considerarse interesante para juzgar la hipótesis de investigación.

En esta investigación la cuenca del río Humuya en Guacamaya queda como representativa para la zona central del país y la de Cayetano para la zona oriental. Los resultados obtenidos en los rendimiento muestran que ambas zonas tienen un rendimiento que cae entre 500 y 1000 ton $/$ año $/ \mathrm{km}^{2}$.

FIGURA 5: Comparación de relaciones $Q S=f(Q L)$

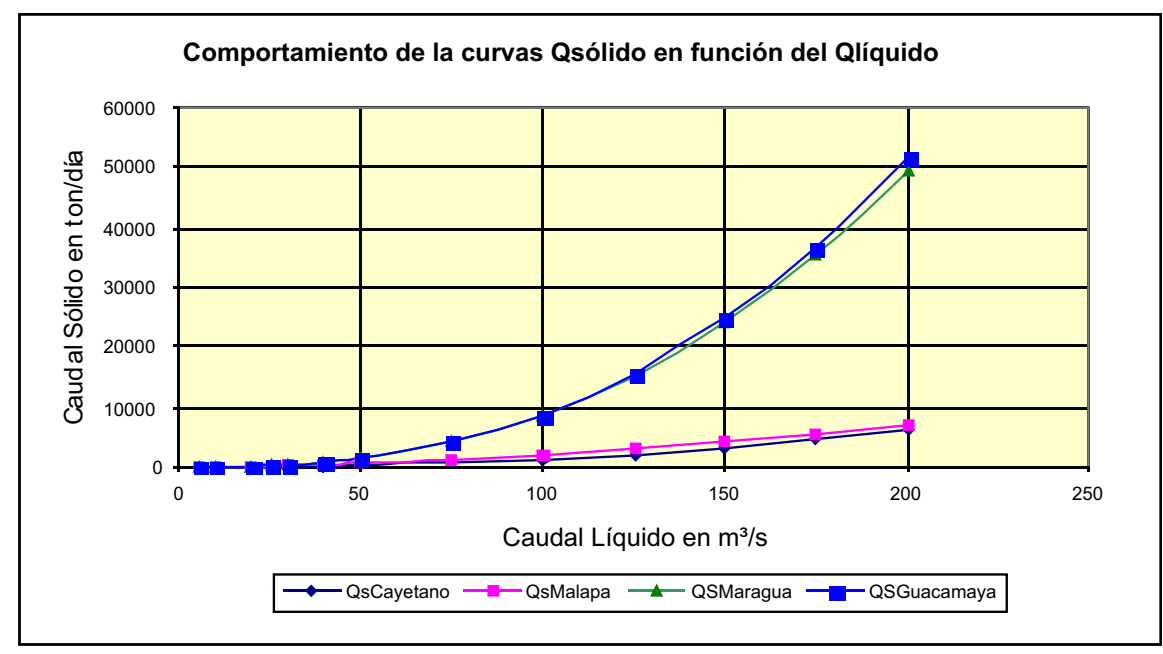

\section{CONCLUSIONES}

De los resultados obtenidos y de su interpretación se concluye lo siguiente:

1. Los datos disponibles de sedimento obtenido por muestreo integral, de las estaciones analizadas en la presente investigación, han sido muestreadas en su mayor parte durante los meses secos. De igual forma la frecuencia de muestreo y su cantidad es variable en cada estación hidrométrica.

2. La extrapolación hacia años anteriores de las relaciones $Q S=f(Q L)$ no son completamente válidas. Porque el sedimento no dependen únicamente del caudal líquido sino también de otras variables, a mencionar como ejemplo suelo y cobertura vegetal. 
3. Las cuencas de los ríos Malapa y Maragua necesitan una mayor cantidad de información para analizar sus rendimientos y pérdida de suelo.

4. De acuerdo al objetivo, a la cantidad y calidad de información disponible durante el desarrollar de la investigación y aceptando que las extrapolaciones favorecen en disponer de una mayor cantidad de información sin asegurar su trascendencia en el tiempo y que el número de muestra es pequeño, se concluye que la hipótesis planteada es aceptada; es decir, que los rendimiento de sedimento de la zona central y oriental del país se encuentran entre $500 \mathrm{y}$ 1000 ton/año/km².

\section{AGRADECIMIENTO}

Se agradece a la Empresa Nacional de Energía Eléctrica del país por haber proporcionado la información de sedimento para las estaciones utilizadas en esta investigación; sin cuya información hubiera sido imposible llevarla acabo.

\section{BIBLIOGRAFÍA}

Avalos Lingan, R. Análisis y Producción de Sedimento para el Proyecto Patuca 3. Tegucigalpa: ENEE; 2007.

Honduras. Secretaría de Recursos Naturales y Ambiente. Dirección General de Recursos Hídricos. Balance Hídrico de Honduras. Tegucigalpa: 2004.

Honduras. ENEE y Consultora GERACON. Estudio de Factibilidad Proyecto Patuca 3. Tegucigalpa: 1997.

Honduras. ENEE y Consultora LAHMEYER. Estudio de Factibilidad de los Proyectos Naranjito y Remolino Sico. Tegucigalpa: 1991.

Honduras. Información de Caudales diarios y los Sedimentos diarios de las estaciones Malapa, Maragua, Guacamaya y Cayetano.

Honduras, ENEE y Motor Columbus, Proyecto El Cajón. Estudio de Factibildad. Volumen 2. Tegucigalpa. Oct. 1973. 